\title{
AN EXTENSION PROBLEM FOR FUNCTIONS WITH MONOTONIC DERIVATIVES
}

\author{
W. B. JURKAT
}

Introduction. This paper deals with questions of the following type. Problem (A): Let $F(x)$ be the $n$th integral of a positive non-decreasing function for all large positive $x$, the problem is to find a function $f(x)$, being the $n$th integral of a non-decreasing function for all $x(-\infty<x<\infty)$, with the property

$$
f(x)= \begin{cases}F(x), & \text { for all large positive } x \\ 0, & \text { for all large negative } x\end{cases}
$$

Problem (A) can be considered as a special case of the boundary value problems, which we discuss in $\S 2$. Roughly speaking, the question is here what values may be assumed by the $n$th integral of a monotonic function and its first $n$ derivatives at the boundary of an interval. It is no loss of generality to suppose the left-hand boundary values to be equal to zero as can be seen by subtracting a suitable polynomial. Then the solution of the problem directly depends on the solution of the reduced Hausdorff and Stieltjes moment problems, for the latter of which we give a new approach ( $\$ 1)$.

The method indicated leads in a simple manner to a complete solution of problem (A), depending on the behaviour of certain quadratic forms (\$3). The main result of the paper consists of determining this behaviour for a large class of functions $F(x)$, for which therefore problem (A) can be settled (Theorem 6).

1. Some reduced moment problems. If a finite sequence $\mu_{\nu}(\nu=0, \ldots, n)$ is given, the reduced Hausdorff moment problem is to determine a nondecreasing function $\psi(t), 0 \leqslant t \leqslant 1$, such that

$$
\mu_{\nu}=\int_{0}^{1} t^{\nu} d \psi(t) \quad(\nu=0, \ldots, n) .
$$

The following result is essentially due to Achyeser-Krein (1; see also 3; 5, pp. $29^{-30} ; 6$, p. 77).

THEOREM 1. A necessary and sufficient condition that the moment problem (I) should have a solution is that, in case $n=2 m$, both quadratic forms

$$
\sum_{i, j=0}^{m} \mu_{i+j} x_{i} x_{j}, \quad \sum_{i, j=0}^{m-1}\left(\mu_{i+j+1}-\mu_{i+j+2}\right) x_{i} x_{j}
$$

Received March 4, 1955. This research was supported by the United States Air Force, through the Office of Scientific Research of the Air Research and Development Command, under Contract Number AF 18 (600)-691. 
should be non-negative, whereas in case $n=2 m+1$, both quadratic forms

$$
\sum_{i, j=0}^{m} \mu_{i+j+1} x_{i} x_{j}, \quad \sum_{i, j=0}^{m}\left(\mu_{i+j}-\mu_{i+j+1}\right) x_{i} x_{j}
$$

should be non-negative.

If a finite sequence $\mu_{\nu}(\nu=0, \ldots, n)$ is given, we also consider the reduced moment problem of determining a non-decreasing function $\psi(t), 0 \leqslant t \leqslant T$, such that

$$
\mu_{\nu}=\int_{0}^{T} t^{\nu} d \psi(t)
$$

with suitable $T>0$.

If (II) has a solution for a certain $T=T_{0}$, then it also has for all $T \geqslant T_{0}$.

Replacing $\mu_{\nu}$ by $\mu_{\nu} / T^{\nu}$ and $x_{i} / T^{i}$ by $x_{i}$ in Theorem 1 we obtain at once

Theorem 2. A necessary and sufficient condition that the moment problem (II) should have a solution is that for $n=2 m$ or $n=2 m+1$ respectively

$$
\sum_{i, j=0}^{m} \mu_{i+j} x_{i} x_{j} \geqslant 0, \quad \sum_{i, j=0}^{m-1} \mu_{i+j+2} x_{i} x_{j} \leqslant T \sum_{i, j=0}^{m-1} \mu_{i+j+1} x_{i} x_{j}
$$

or

$$
\sum_{i, j=0}^{m} \mu_{i+j+1} x_{i} x_{j} \geqslant 0, \quad \sum_{i, j=0}^{m} \mu_{i+j+1} x_{i} x_{j} \leqslant T \sum_{i, j=0}^{m} \mu_{i+j} x_{i} x_{j}
$$

should hold for all values of $x_{i}$ and a suitable $T>0$.

Corollary. A sufficient condition is that for $n=2 m$ or $n=2 m+1$ respectively the quadratic forms

$$
\sum_{i, j=0}^{m} \mu_{i+j} x_{i} x_{j}, \quad \sum_{i, j=0}^{m-1} \mu_{i+j+1} x_{i} x_{j}
$$

or

$$
\sum_{i, j=0}^{m} \mu_{i+j+1} x_{i} x_{j}, \quad \sum_{i, j=0}^{m} \mu_{i+j} x_{i} x_{j}
$$

should be positive definite, whereas a necessary condition is that they should be non-negative.

It is worthwhile to point out the close connection between problem (II) and the reduced Stieltjes moment problem of determining a non-decreasing function $\psi(t), 0 \leqslant t<\infty$, such that

$$
\mu_{\nu}=\int_{0}^{\infty} t^{\nu} d \psi(t) \quad(\nu=0, \ldots, n) .
$$

Necessary and sufficient conditions for the moment problem (III) to have a solution are due to Verblunsky (7), who based his argument on certain algebraic lemmas of $\mathrm{E}$. Fischer. By means of Theorem 1 or 2 we are able to give a new and very simple approach, avoiding with Verblunsky the theory of 
continued fractions. This approach will also give us more detailed information about the solution.

First we infer from Theorem $13 \mathrm{~b}$ of Widder (8, p. 138) that a necessary and sufficient condition that the moment problem (III) should have a solution $\psi(t)$ with infinitely many points of increase is that the quadratic forms (5) resp. (6) should be positive definite (cf 8, p. 6). The necessary part is trivial and the sufficient part follows by inductive definition of $\mu_{n+1}, \mu_{n+2}, \ldots$.

Now the Corollary to Theorem 2 shows that, if there is a solution of (III) with infinitely many points of increase, then there also is a solution of (II). If there is a solution of (III) with a finite number of points of increase (that is, a step-function with finitely many steps) the same conclusion is true. Conversely every solution of (II) also gives a solution of (III), such that the problems (II) and (III) are equivalent. The conditions of Theorem 2, now also valid for problem (III), are slightly different from those of Verblunsky and have the advantage of using only the known values $\mu_{0}, \ldots, \mu_{n}$.

With the help of a mean value theorem for systems of integrals (4, p. 97) we further see that, if there is a solution of (II), then there also is a solution of (II) by a non-decreasing step-function with finitely many steps. Hence, if (III) has a solution, then (III) even has a solution with a finite number of points of increase. Therefore the conditions of Theorem 2 also are necessary and sufficient for the moment problem (III) to have a solution $\psi(t)$ with a finite number of points of increase.

Similar arguments can be used for the reduced Hamburger moment problem

$$
\mu_{\nu}=\int_{-\infty}^{\infty} t^{\nu} d \psi(t) \quad(\nu=0, \ldots, n) .
$$

2. Some boundary value problems for functions with monotonic derivatives. We consider the following problem (B): Given real numbers $c_{\nu}(\nu=0, \ldots, n), X$, and $T>0$, find a function $f(x)$, which is for $X-T \leqslant$ $x \leqslant X$ the $n$th integral of a non-decreasing function $\phi(x)$ and satisfies the boundary value condition

$$
f^{(\nu)}(X-T)=0, \quad f^{(\nu)}(X)=c_{\nu} \quad(\nu=0, \ldots, n),
$$

where $f^{(n)}(x)$ is to be identified with $\phi(x)$ by definition. (For points of continuity $f^{(n)}(x)=\phi(x)$ holds by itself, and for other points $f^{(n)}(x), n \geqslant 1$, is not defined a priori.) Besides (B) we introduce the problem ( $\left.\mathrm{B}^{\prime}\right)$ differing from (B) only in the possibility that $T>0$ may be chosen suitably.

If problem (B) or $\left(\mathrm{B}^{\prime}\right)$ has a solution, then for $X-T \leqslant x \leqslant T$ and $\nu=0, \ldots, n$ we have

$$
f^{(\nu)}(x)=\frac{1}{(n-\nu) !} \int_{X-T}^{x}(x-t)^{n-\nu} d \phi(t)
$$

in particular,

$$
c_{\nu}=\frac{1}{(n-\nu) !} \int_{X-T}^{X}(X-t)^{n-\nu} d \phi(t) .
$$


Conversely, if (8) holds with a non-decreasing function $\phi(x)$, where we may. assume $\phi(X-T)=0$ without restriction, then

$$
f(x)=\frac{1}{n !} \int_{X-T}^{x}(x-t)^{n} d \phi(t)
$$

is a solution of $(\mathrm{B})$ or $\left(\mathrm{B}^{\prime}\right)$.

By a change of variables the condition (8) can be written in the form

$$
\nu ! c_{n-\nu}=\int_{0}^{T} t^{\nu} d \psi(t) \quad(\nu=0, \ldots, n),
$$

or

$$
\nu ! c_{n-\nu} / T^{\nu}=\int_{0}^{1} t^{\nu} d \psi(t) \quad(\nu=0, \ldots, n),
$$

with non-decreasing functions $\psi(t)$.

Thus we have proved the following results:

Theorem 3. Problem (B) has a solution if and only if problem (I) has a solution with

$$
\mu_{\nu}=\nu ! c_{n-\nu} / T^{\nu} \quad(\nu=0, \ldots, n) .
$$

Theorem 4. Problem ( $\left.\mathrm{B}^{\prime}\right)$ has a solution if and only if problem (II) has a solution with

$$
\mu_{\nu}=\nu ! c_{n-\nu} \quad(\nu=0, \ldots, n) .
$$

Explicit conditions can be taken from Theorems 1 and 2 .

3. Extension theorems for functions with monotonic derivatives. We now consider problem (A) at the beginning of this paper. A simple argument shows:

THEOREM 5. Problem (A) has a solution if and only if problem ( $\mathrm{B}^{\prime}$ ) has a solution with

$$
c_{\nu}=F^{(v)}(X) \quad(\nu=0, \ldots, n)
$$

for all large positive $X\left(X\right.$ only denoting numbers, where $F^{(n)}(X)$ exists $)$.

Explicit conditions can be taken from Theorem 2 by means of Theorem 4 . We shall only use the special conditions of the Corollary of Theorem 2:

Corollary. A sufficient (necessary) condition that problem (A) should have $a$ solution is that for $n=2 m$ or $n=2 m+1$ respectively the quadratic forms

$$
\sum_{i, j=0}^{m}(i+j) ! F^{(n-i-j)}(X) x_{i} x_{j}, \quad \sum_{i, j=0}^{m-1}(i+j+1) ! F^{(n-i-j-1)}(X) x_{i} x_{j}
$$

or

$$
\sum_{i, j=0}^{m}(i+j+1) ! F^{(n-i-j-1)}(X) x_{i} x_{j}, \quad \sum_{i, j=0}^{m}(i+j) ! F^{(n-i-j)}(X) x_{i} x_{j}
$$


should be positive definite (non-negative) for all large positive $X$ ( $X$ only denoting numbers, where $F^{(n)}(X)$ exists).

In general it is rather difficult to decide whether the forms (12), (13) are positive definite or not. But there is a certain class of functions $F(x)$ for which we can give a complete solution. These are the $L$-functions or logarithmico-exponential functions in the sense of $\operatorname{Hardy}(2$, p. 17).

Theorem 6. Necessary and sufficient that the problem (A) should have a solution for a L-function $F(x)$ and $n \geqslant 2$ is that $F(x) / x^{n}$ should be non-decreasing for all large positive $x$. For $n=0,1$ there is always a solution.

Proof. The existence of a solution for $n=0,1$ follows at once from the Corollary and the fact that $F^{(n)}(X), F^{(n-1)}(X)$ are positive for $X \rightarrow+\infty$. From now on we may assume $n \geqslant 2$. Using the elementary properties of $L$-functions and our supposition on $F(x)$,

$$
F^{(n+1)}(x) \geqslant 0, \quad F^{(n)}(x) \geqslant \epsilon>0, \quad x \rightarrow+\infty,
$$

we only have to discuss the following cases:

$$
x F^{(n+1)}(x) \geqslant \delta>0,
$$$$
x \rightarrow+\infty
$$

or

$$
F(x)=\frac{x^{n}}{n !} L(x), \quad 0<\delta \leqslant L(x)=o(\log x), \quad x \rightarrow+\infty,
$$

with the possibilities, for $x \rightarrow+\infty$,

$$
\left(b_{1}\right) \quad L^{\prime}(x)=0, \quad\left(b_{2}\right) \quad L^{\prime}(x)>0, \quad\left(b_{3}\right) \quad L^{\prime}(x)<0 .
$$

We shall show that (A) has a solution in cases $(a),\left(b_{1}\right),\left(b_{2}\right)$, where $F(x) / x^{n}$ is non-decreasing for $x \rightarrow+\infty$, and that (A) has no solution in case $\left(b_{3}\right)$, where $F(x) / x^{n}$ is strictly decreasing for $x \rightarrow+\infty$. All this together will prove the Theorem.

Example. If $F(x)=x^{n}+x^{n-1}$, problem (A) has a solution for $n=1$, but no solution for $n \geqslant 2$.

In case (a) we use the sufficient part of the Corollary and restrict ourselves to the form

$$
\sum_{i, j=0}^{m} \frac{(i+j) !}{X^{i+j}} F^{(n-i-j)}(X) x_{i} x_{j},
$$

where $x_{i} X^{i}$ is replaced by $x_{i}$. We assume that (15) has its minimum value $M(X)$ on $\Sigma x_{i}{ }^{2}=1$ for $x_{i}=x_{i}(X)$. It is enough to show that $M(X)>0$ holds for $X \rightarrow+\infty$, or for any sequence $X=X_{k} \rightarrow \infty$ such that $x_{i}\left(X_{k}\right) \rightarrow \xi_{i}$ for $i=0, \ldots, m$, with $\Sigma \xi_{i}{ }^{2}=1$.

For $\nu=0, \ldots, n$ and $X \geqslant x_{0}\left(x_{0}\right.$ large enough) we have

$$
F^{(n-\nu)}(X)=\frac{1}{\nu !} \int_{x_{0}}^{X}(X-x)^{\nu} F^{(n+1)}(x) d x+\sum_{\zeta=0}^{\nu} \frac{\left(X-x_{0}\right)^{\zeta}}{\zeta !} F^{(n-\nu+\zeta)}\left(x_{0}\right)
$$


and therefore

$$
\begin{aligned}
M(X) & =\int_{x_{0}}^{X}\left(\sum_{i=0}^{m} \frac{(X-x)^{i}}{X^{i}} x_{i}\right)^{2} F^{(n+1)}(x) d x \\
& +F^{(n)}\left(x_{0}\right)\left(\sum_{i=0}^{m} \frac{\left(X-x_{0}\right)^{i}}{X^{i}} x_{i}\right)^{2}+O\left(X^{-1}\right) .
\end{aligned}
$$

Hence, taking $X=X_{k}$ and using (a),

$$
\begin{aligned}
M(X) & \geqslant \frac{\delta}{X} \int_{x_{0}}^{X}\left(\sum_{i=0}^{m} \frac{(X-x)^{i}}{X^{i}} x_{i}\right)^{2} d x+O\left(X^{-1}\right) \\
& \geqslant \frac{\delta}{X} \sum_{i, j=0}^{m} \frac{\left(X-x_{0}\right)^{i+j+1}}{i+j+1} \frac{x_{i} x_{j}}{X^{i+j}}+O\left(X^{-1}\right) \\
& \geqslant \delta \sum_{i, j=0}^{m} \frac{\xi_{i} \xi_{j}}{i+j+1}+o(1) \\
& \geqslant \delta \int_{0}^{1}\left(\sum_{i=0}^{m} \xi_{i} x^{i}\right)^{2} d x+o(1) .
\end{aligned}
$$

Since

$$
\int_{0}^{1}\left(\sum_{i=0}^{m} \xi_{i} x^{i}\right)^{2} d x>0, \quad \sum \xi_{i}{ }^{2}=1
$$

we obtain $M\left(X_{k}\right)>0$ for $X_{k} \rightarrow+\infty$.

The same method can be used to show that the other forms in the Corollary are positive definite also.

In case $\left(b_{1}\right), F(x)=c x^{n}$ holds for $x \rightarrow+\infty$ with positive $c$. Then a solution of the problem (A) is given by the function

$$
f(x)= \begin{cases}c x^{n}, & x \geqslant 0 \\ 0, & x \leqslant 0 .\end{cases}
$$

In case $\left(b_{2}\right)$, instead of $(16)$ we use the Leibniz formula

$$
\frac{\nu !}{X^{\nu}} F^{(n-\nu)}(X)=L(X)+\sum_{\zeta=1}^{n-\nu}\left(\begin{array}{c}
n-\nu \\
\zeta
\end{array}\right) L^{(\zeta)}(X) \frac{X^{\nu+\zeta}}{(\nu+\zeta) !} \frac{\nu !}{X^{\nu}}
$$

for $\nu=0, \ldots, n$ and $X \rightarrow+\infty$, and the asymptotic formulae

$$
X L^{\prime}(X)=o(L(X))
$$

and

$$
X^{\zeta} L^{(\zeta)}(X)=(-1)^{\zeta-1}(\zeta-1) ! X L^{\prime}(X)+o\left(X L^{\prime}(X)\right)
$$

for $\zeta=1,2, \ldots$ and $X \rightarrow+\infty$, which are consequences of (b) and Hardy (2, p. 37, line 5). From both together it follows for $\nu=0, \ldots, n$ and $X \rightarrow+\infty$ that

$$
\frac{\nu !}{X^{\nu}} F^{(n-\nu)}(X)=L(X)+\gamma_{\nu} X L^{\prime}(X)+o\left(X L^{\prime}(X)\right)
$$


where

$$
\left\{\begin{aligned}
\gamma_{\nu} & =\sum_{\zeta=1}^{n-\nu}\left(\begin{array}{c}
n-\nu \\
\zeta
\end{array}\right)(-1)^{\zeta-1} \frac{\nu !(\zeta-1) !}{(\nu+\zeta) !} & \\
& =\sum_{\zeta=1}^{n-\nu}\left(\begin{array}{c}
n-\nu \\
\zeta
\end{array}\right)(-1)^{\zeta-1} \int_{0}^{1}(1-x)^{\nu} x^{\zeta-1} d x & \\
& =\int_{0}^{1} \frac{(1-x)^{\nu}-(1-x)^{n}}{x} d x, & \nu=0, \ldots, n .
\end{aligned}\right.
$$

Hence

$$
\begin{aligned}
M(X) & =L(X)\left(\sum_{i=0}^{m} x_{i}\right)^{2}+o\left(X L^{\prime}(X)\right) \\
& +X L^{\prime}(X) \int_{0}^{1}\left\{\left(\sum_{i=0}^{m} x_{i}(1-x)^{i}\right)^{2}-(1-x)^{n}\left(\sum_{i=0}^{m} x_{i}\right)^{2}\right\} \frac{d x}{x} .
\end{aligned}
$$

Now let

$$
X=X_{k}, \quad \sum_{i=0}^{m} \xi_{i} \neq 0
$$

Then

$$
M(X)=L(X)\left(\sum_{i=0}^{m} \xi_{i}\right)^{2}+o(L(X)),
$$

and on account of (b) we get $M\left(X_{k}\right)>0$ for $X_{k} \rightarrow+\infty$. In the remaining case

$$
\sum_{i=0}^{m} \xi_{i}=0
$$

we have for $X=X_{k}$

$$
M(X) \geqslant X L^{\prime}(X) \int_{0}^{1}\left(\sum_{i=0}^{m} \xi_{i}(1-x)^{i}\right)^{2} \frac{d x}{x}+{ }^{\prime \prime} o\left(X L^{\prime}(X)\right) .
$$

Since

$$
\int_{0}^{1}\left(\sum_{i=0}^{m} \xi_{i}(1-x)^{i}\right)^{2} \frac{d x}{x}>0, \quad \sum_{i=0}^{m} \xi_{i}=0, \quad \sum_{i=0}^{m} \xi_{i}{ }^{2}=1,
$$

we find $M\left(X_{k}\right)>0$ for $X_{k} \rightarrow+\infty$. The same method can be used to show that the other forms in the Corollary are positive definite also.

In case $\left(b_{3}\right)$ we use the necessary part of the Corollary and show that the minimum $M(X)$ of the form (15) on $\Sigma x_{i}{ }^{2}=1$ is negative for $X \rightarrow+\infty$. Taking $\xi_{i}$ with

$$
\sum_{i=0}^{m} \xi_{i}=0, \quad \sum_{i=0}^{m} \xi_{i}^{2}=1
$$

we obtain similarly to (22) and (23) 


$$
\begin{aligned}
M(X) & \leqslant \sum_{i, j=0}^{m} \frac{(i+j) !}{X^{i+j}} F^{(n-i-j)}(X) \xi_{i} \xi_{j} \\
& \leqslant X L^{\prime}(X) \int_{0}^{1}\left(\sum_{i=0}^{m} \xi_{i}(1-x)^{i}\right)^{2} \frac{d x}{x}+o\left(X L^{\prime}(X)\right) \\
& <0, \quad X \rightarrow+\infty .
\end{aligned}
$$

This completes the proof of Theorem 6 .

\section{REFERENCES}

1. N. Achyeser and M. Krein. Über eine Transformation der reellen Toeplitzschen Formen und das Momentenproblem in einem endlichen Intervalle, Commun. Soc. Math. Kharkoff (4), 11 (1935), 21-26.

2. G. H. Hardy, Orders of infinity (Cambridge Tract, 1924).

3. L. Kantorovič, On the moment problem for a finite interval, C. R. (Doklady), Acad. Sci. URSS (N.S.), 14 (1937), 531-537.

4. G. Kowalewski, Integralgleichungen (Berlin, 1930).

5. M. Krein, The ideas of P.L. Čebyšev and A. A. Markov in the theory of limiting values of integrals and their further development, Upsekhi Matem. Nauk (N.S.) 6 (1951), 3-120.

6. J. A. Shohat and J. D. Tamarkin, The problems of moments (New York, 1943).

7. S. Verblunsky, On a problem of moments, Proc. Cambridge Philos. Soc. 45 (1949), 1-4.

8. D. V. Widder, The Laplace transform (Princeton, 1946).

\section{University of Cincinnati}

